\title{
Neurodevelopmental Outcome in High-Risk Congenital Diaphragmatic Hernia Patients: An Appeal for International Standardization
}

\author{
Kitty G. Snoek ${ }^{a} \quad$ Irma Capolupo ${ }^{c}$ Annabella Bragugliac Lucia Aite ${ }^{c}$ \\ Joost van Rosmalen ${ }^{b}$ Laura Valfrèc René M. Wijnen ${ }^{\mathrm{a}}$ Pietro Bagolan ${ }^{c}$ \\ Dick Tibboel $^{a}$ Hanneke IJsselstijn $^{a}$ \\ antensive Care and Department of Pediatric Surgery, Erasmus MC-Sophia Children's Hospital, and ${ }^{\text {b Department }}$

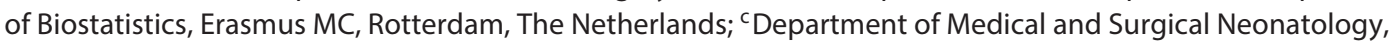 \\ Bambino Gesu Children's Hospital, Rome, Italy
}

\section{Key Words}

Congenital diaphragmatic hernia · Follow-up program .

Neurodevelopmental outcome

\begin{abstract}
Background: Since mortality in congenital diaphragmatic hernia $(\mathrm{CDH})$ is decreasing, morbidity such as neurodevelopmental outcome is becoming increasingly important. Objectives: We evaluated neurodevelopmental outcome in high-risk $\mathrm{CDH}$ patients treated according to the $\mathrm{CDH}$ EURO Consortium standardized treatment protocol. Methods: This observational, prospective cohort study was conducted in two European centers. Neurodevelopment of 88 patients (Rotterdam $n=49$; Rome $n=39$ ) was assessed at 12 and 24 months with the Bayley Scales of Infant Development (BSID)-II-NL (Rotterdam) or BSID-III (Rome). Data of the centers were analyzed separately. Results: Cognition was normal in $77.8 \%$ of children from Rotterdam and in $94.8 \%$ from Rome at 12 months, and in 70.7 and $97.4 \%$, respectively, at 24 months. Motor function was normal in $64.3 \%$ from Rotterdam and in $81.6 \%$ from Rome at 12 months and in 45.7 and $89.8 \%$, respectively, at 24 months. Longer length of hospital stay (LoS) was associated with worse cognitive outcome and motor function; LoS, low socioeconomic sta-
\end{abstract}

tus, and ethnicity were associated with lower cognition. Conclusions: At 2 years, most CDH patients have normal cognition, but are at risk for motor function delay. Due to differences in outcomes between centers, careful interpretation is needed before conclusions can be drawn for other centers. Future multicenter collaboration should not only focus on standardization of postnatal care, but also on international standardization of follow-up to identify risk factors and thereby reduce morbidity.

(c) 2015 S. Karger AG, Basel

\section{Introduction}

Congenital diaphragmatic hernia $(\mathrm{CDH})$ affects approximately 1 in 3,000 newborns [1]. It is a life-threatening disease caused mainly by persistent pulmonary hypertension and pulmonary hypoplasia [1]. Severely ill $\mathrm{CDH}$ patients may receive extracorporeal membrane oxygenation (ECMO) [1]. In 2008, a standardized neonatal treatment protocol was developed at a consensus meeting of the CDH EURO Consortium [1]. The mortality rate has decreased from 33 to $12 \%$ since this protocol was implemented [2]. Consequently, worldwide the focus of interest has shifted to morbidity [3-5].

\section{KARGER}

E-Mail karger@karger.com

www.karger.com/neo
(C) 2015 S. Karger AG, Base

1661-7800/15/1091-0014\$39.50/0
Hanneke IJsselstijn, MD, PhD

Intensive Care and Department of Pediatric Surgery, Room Sk-1280

Erasmus MC-Sophia Children's Hospital, PO Box 2060

NL-3000 CB, Rotterdam (The Netherlands)

E-Mail h.ijsselstijn@erasmusmc.nl 


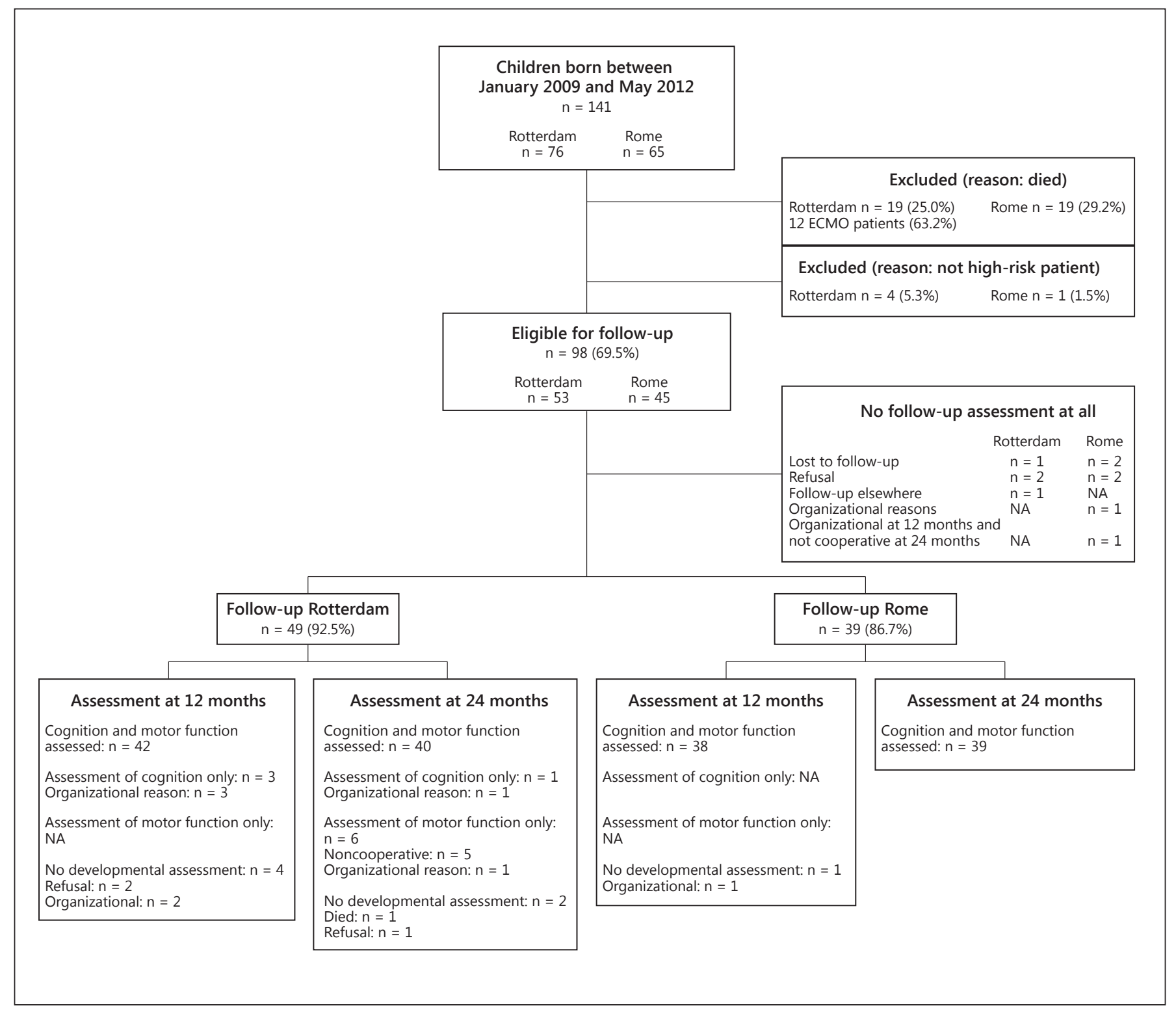

Fig. 1. Flowchart of inclusion of all patients. NA = Not applicable.

Previous studies found associated morbidity in about $87 \%$ of $\mathrm{CDH}$ survivors, mainly related to the lungs or the gastrointestinal tract $[6,7]$. While most follow-up studies have focused on morbidity, few have examined neurodevelopment. Most of those studies were cross-sectionally performed in small series in single centers [8-10]. Recently, longitudinal studies have shown neurodevelopmental delays in $\mathrm{CDH}$ patients $[3,4,11]$. To date, no published studies have examined neurodevelopmental outcome after implementation of a standardized treatment protocol in a multicenter setting.

Neurodevelopmental Outcome in $\mathrm{CDH}$
We longitudinally investigated neurodevelopmental outcome in high-risk patients managed according to the CDH EURO Consortium standardized treatment protocol [1] in two high-volume CDH centers. Secondly, we identified determinants of neurodevelopmental outcome.

\section{Materials and Methods}

This is an observational, prospective cohort study in patients born between January 2009 and May 2012 with high-risk CDH, i.e. antenatal diagnosis/respiratory insufficiency within $6 \mathrm{~h}$ postnatal- 
ly. All patients were treated according to a standardized treatment protocol [1]; however, ECMO was only offered in Rotterdam. Rotterdam and Rome are two of the largest European CDH centers. As standard of care, survivors were offered a structured, longitudinal follow-up program, initiated in Rotterdam in 1999 [11] and in Rome in 2004 [12]. We evaluated prospectively collected data of repeated measurements at corrected ages of 12 and 24 months. Since subjects were not submitted to any handling and no rules of human behavior were imposed, institutional review board approval was waived. Parents were informed that data were used for research purposes.

Patient characteristics were retrieved from medical records. Neurodevelopmental outcome was assessed with the Bayley Scales of Infant Development (BSID). In Rotterdam, the Dutch-language version of the BSID-II (BSID-II-NL) was administered with Dutch normative data [13]. In both centers cognitive development was assessed at both time points (by two developmental psychologists per center). Psychomotor development was assessed by developmental psychologists at both time points in Rome and at 12 months in Rotterdam. In Rotterdam, psychomotor development was assessed by one physiotherapist at 24 months. In Rome, the Italian translation of the BSID-III [14] with American normative data was administered. Scores were grouped as normal ( $>-1$ SD), mildly delayed $(-2<\mathrm{SD}<-1)$ and severely delayed $(<-2 \mathrm{SD})$. For further details, see the supplementary file Methods (for all suppl. material, see www.karger.com/doi/10.1159/000438978).

\section{Data Analysis}

Descriptive statistics are presented as number (\%), mean (SD) or median (range). Independent samples t tests, $\chi^{2}$ tests and MannWhitney $U$ tests, where appropriate, were used to compare characteristics between centers. One-sample t tests served to compare mean scores to the normalized population mean. Linear mixed models were used to estimate neurodevelopmental outcome over time. These models included only the time point ( 12 or 24 months) as the independent variable, and a random intercept to account for the within-subject correlations. Perinatal factors associated with adverse neurodevelopment were determined using multivariate linear regression. Cognition and motor outcome were chosen as dependent variables in two different models; the data were analyzed separately for each assessment, age and each center. Based on clinical experience and the literature, the following independent variables were included in the linear regression analysis: tube feeding at discharge, ECMO support, ethnicity and SES as categorical variables, and SNAP-II score and length of hospital stay (LoS; logtransformed due to skewed distribution) as continuous variables. Analyses were performed using SPSS version 20.

\section{Results}

In the study period, $141 \mathrm{CDH}$ patients were admitted (Rotterdam $\mathrm{n}=76$; Rome $\mathrm{n}=65$ ). Thirty-eight children died (Rotterdam $\mathrm{n}=19$; Rome $\mathrm{n}=19$ ) and 5 were not high-risk $\mathrm{CDH}$ patients; thus, 98 children were eligible for follow-up. Ultimately, 88 patients were assessed (fig. 1).
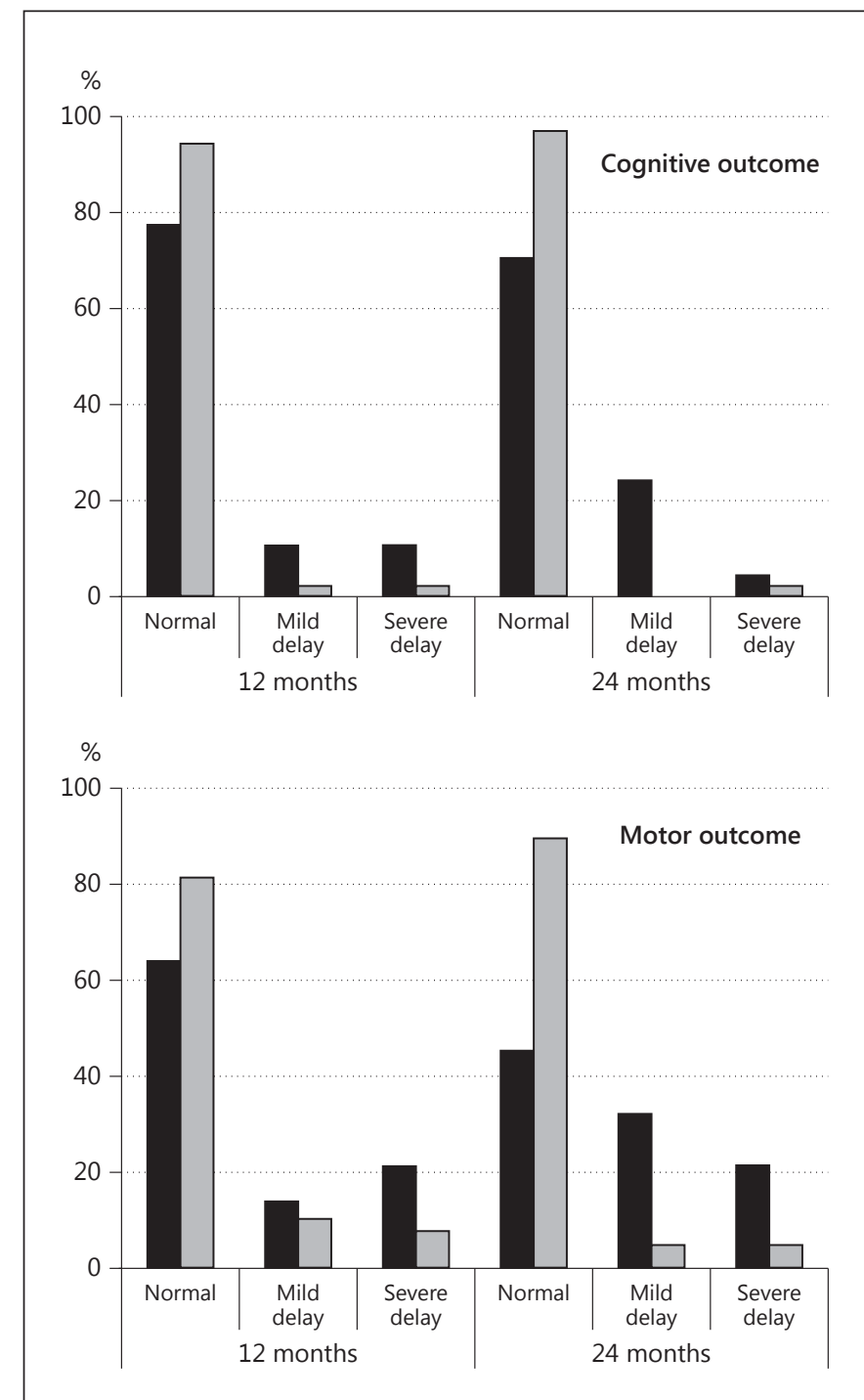

Fig. 2. Neurodevelopmental outcome in $\mathrm{CDH}$ survivors at 12 and 24 months. Black: Rotterdam; grey: Rome.

Baseline characteristics of participants (table 1) were not significantly different from nonparticipants. Four patients (Rotterdam $\mathrm{n}=1$; Rome $\mathrm{n}=3$ ) underwent fetoscopic endotracheal occlusion. In Rotterdam, 28 patients underwent thoracoscopic repair and 7 were converted to laparotomy. In Rome, all children underwent laparotomy. None of the children received anticonvulsants. Six patients from Rotterdam had a genetic or chromosomal abnormality that was not associated with neurodevelopment. Patch repair, episodes of general anesthesia 0-24 months, and involvement of a physical therapist and speech-language pathologist were significantly different
16 
Table 1. Background characteristics

\begin{tabular}{|c|c|c|c|}
\hline & $\begin{array}{l}\text { Rotterdam } \\
(\mathrm{n}=49)\end{array}$ & $\begin{array}{l}\text { Rome } \\
(\mathrm{n}=39)\end{array}$ & $\mathrm{p}$ value $^{1}$ \\
\hline \multicolumn{4}{|c|}{ Perinatal and postnatal characteristics } \\
\hline LHR & $1.9(0.8)$ & $2.2(0.8)$ & 0.32 \\
\hline Missing & 15 & 13 & \\
\hline O/E LHR & $51.6(17.4)$ & $53.3(15.8)$ & 0.71 \\
\hline Missing & 21 & 13 & \\
\hline Male sex & $28(57.1 \%)$ & $25(64.1 \%)$ & 0.51 \\
\hline Birth weight, kg & $3.07(0.56)$ & $2.99(0.41)$ & 0.45 \\
\hline $\begin{array}{l}\text { Born before } 37 \text { weeks of } \\
\text { gestation }\end{array}$ & $8(16.3 \%)$ & $4(10.3 \%)$ & 0.41 \\
\hline SNAP-II score & $18(12)$ & $13(9)$ & 0.07 \\
\hline Age at repair, days & $3.8(2.7)$ & $3.6(2.1)$ & 0.77 \\
\hline Left-sided CDH & $42(85.7 \%)$ & $38(97.4 \%)$ & 0.06 \\
\hline Liver: intrathoracic & $19(38.8 \%)$ & $12(30.8 \%)$ & 0.44 \\
\hline Defect size & & & 0.24 \\
\hline A & $5(10.2 \%)$ & $4(10.3 \%)$ & \\
\hline $\mathrm{B}$ & $18(26.7 \%)$ & $22(56.4 \%)$ & \\
\hline $\mathrm{C}$ & $24(49.0 \%)$ & $11(28.2 \%)$ & \\
\hline $\mathrm{D}$ & $2(4.1 \%)$ & $2(5.1 \%)$ & \\
\hline Patch repair & $35(71.4 \%)$ & $11(28.2 \%)$ & $<0.001$ \\
\hline Defect size A & $\mathrm{n}=1$ & $\mathrm{n}=0$ & \\
\hline Defect size B & $\mathrm{n}=8$ & $\mathrm{n}=1$ & \\
\hline Defect size C & $\mathrm{n}=24$ & $\mathrm{n}=8$ & \\
\hline Defect size D & $\mathrm{n}=2$ & $\mathrm{n}=2$ & \\
\hline Initial ventilation & & & 0.83 \\
\hline CMV & $24(49.0 \%)$ & $20(51.3 \%)$ & \\
\hline $\mathrm{HFO}$ & $25(51.0 \%)$ & $19(48.7 \%)$ & \\
\hline Length of ventilation, days & $8(1-271)$ & $8(2-70)$ & 0.55 \\
\hline $\begin{array}{l}\text { Length of initial hospital } \\
\text { stay, days }\end{array}$ & $28(6-387)$ & $30(15-161)$ & 0.54 \\
\hline CLD & & & 0.12 \\
\hline No CLD & $29(59.2 \%)$ & $32(82.1 \%)$ & \\
\hline Mild CLD & $8(16.3 \%)$ & $2(5.1 \%)$ & \\
\hline Moderate CLD & $1(2.0 \%)$ & $0(0 \%)$ & \\
\hline Severe CLD & $11(22.4 \%)$ & $5(12.8 \%)$ & \\
\hline
\end{tabular}

between Rotterdam and Rome (table 1). After exclusion of ECMO-treated patients (online suppl. table 1a), episodes of general anesthesia 0-24 months and involvement of physical therapy were not significantly different between the two centers (online supplementary table 1b).

Based on the cutoff scores from the BSID manuals, cognition was normal in 71 children (85.5\%) and motor

Rotterdam Rome

$(\mathrm{n}=49) \quad(\mathrm{n}=39)$

Length of morphinomimetics/sedatives

$\begin{array}{lll}<1 \text { week } & 25(51.0 \%) & 14(35.9 \%) \\ 1 \text { week to 1 month } & 17(34.7 \%) & 20(51.3 \%) \\ >1 \text { month } & 7(14.3 \%) & 5(12.8 \%)\end{array}$

0.28

Episodes of general

anesthesia $0-24$ months $2(1-13) \quad 1(1-3) \quad 0.01$

At discharge

$\begin{array}{llll}\text { Tube feeding } & 29(59.2 \%) & 20(51.3 \%) & 0.46\end{array}$

\begin{tabular}{llll}
\hline Physical therapy at home & $20(40.8 \%)$ & $7(17.9 \%)$ & 0.02
\end{tabular}

Speech language pathologist

involved

$19(38.8 \%) \quad 3(7.7 \%)$

0.001

Demographic variables

Ethnicity

Native $\quad 34(69.4 \%) \quad 33(84.6 \%)$

Nonnative $\quad 14(28.6 \%) \quad 6(15.4 \%)$

Unknown $\quad 1(2.0 \%) \quad 0(0 \%)$

\begin{tabular}{|c|c|c|}
\hline SES mother & & 0.12 \\
\hline Low & $13(26.5 \%)$ & $9(23.1 \%)$ \\
\hline Medium & $15(30.6 \%)$ & $21(53.8 \%)$ \\
\hline High & $18(36.7 \%)$ & $9(23.1 \%)$ \\
\hline Unknown & $3(6.1 \%)$ & $0(0 \%)$ \\
\hline
\end{tabular}

Categorical variables are shown as $\mathrm{n}(\%)$ and compared between centers using $\chi^{2}$ tests, normally distributed variables are shown as means (SD) and compared between groups using independent samples t tests, and continuous variables that are not normally distributed are shown as medians (range) and compared between centers using Mann-Whitney $U$ tests. LHR = Lung-head ratio [15]; O/E LHR = observed to expected lung-head ratio; SNAP-II score $=$ Score for Neonatal Acute Physiology-II [16]; $\mathrm{CMV}=$ conventional mechanical ventilation; $\mathrm{HFO}=$ high-frequency oscillation; CLD = chronic lung disease [17]; SES = socioeconomic status [18]. ${ }^{1}$ Comparison between Rotterdam and Rome.

function was normal in 58 children (72.5\%) at 12 months; this held true for 67 patients $(83.8 \%)$ and 56 patients (65.9\%), respectively, at 24 months. Proportions of mild and severe neurodevelopmental delay are shown in figure 2 . Table 2 shows means (SD) of neurodevelopmental outcome. In online supplementary figure $2 \mathrm{a}$ and online supplementary table $2 \mathrm{a}$, data were presented after exclusion of ECMO-treated patients.

In Rotterdam, cognition and motor function remained stable over time. In Rome, cognition and motor function improved over time [mean differences $4.1(\mathrm{p}=0.02)$ and $5.8(\mathrm{p}=0.003)$, respectively] 
Table 2. Neurodevelopmental outcome scores at 12 and 24 months (corrected for prematurity)

\begin{tabular}{|c|c|c|c|c|c|c|c|c|}
\hline Cognitive outcome $e^{4}$ & $97.8(19.8)$ & 0.46 & $97.9(11.8)$ & 0.28 & $96.0(18.4)$ & 0.17 & $102.1(13.9)$ & 0.36 \\
\hline Motor outcome ${ }^{4}$ & $87.7(18.8)$ & $<0.001$ & $93.2(12.2)$ & 0.002 & $82.9(16.7)$ & $<0.001$ & $98.2(14.8)$ & 0.45 \\
\hline Language outcome $\mathrm{e}^{4}$ & - & - & $97.7(8.6)$ & 0.10 & - & - & $97.7(12.6)$ & 0.26 \\
\hline Fine motor skills ${ }^{5}$ & - & - & $10.1(2.1)$ & 0.76 & - & - & $10.5(2.6)$ & 0.23 \\
\hline Gross motor skills ${ }^{5}$ & - & - & $7.7(2.5)$ & $<0.001$ & - & - & $8.9(2.7)$ & 0.01 \\
\hline
\end{tabular}

${ }^{1}$ Cognitive outcome: $n=45$; motor outcome: $n=42 .{ }^{2}$ Outcome scores were compared to the expected normal score of reference population; Rotterdam: BSID-II-NL; Rome: BSID-III. ${ }^{3}$ Cognitive outcome: $n=41$; motor outcome: $n=46 .{ }^{4}$ Mean $(\mathrm{SD})$ of expected normal score of reference population is 100 (15). ${ }^{5}$ Mean (SD) of expected normal score of reference population is 10 (3).

In Rotterdam, cognitive outcome at 24 months was negatively associated with low socioeconomic status (SES) (B -36.2; 95\% CI: -58.1 to -14.3) and non-Dutch ethnicity (B 25.1; 95\% CI: 5.9 to 44.2; table 3). Motor function was negatively associated with longer LoS at 24 months (B -7.8; 95\% CI: -15.3 to -0.3 ). For example, taking into account the logarithmic transformation of LoS, children who were hospitalized twice as long scored 5.4 points lower (calculation: $\mathrm{B} \times \ln 2=-7.8 \times \ln 2$ ) on motor function at 24 months. In non-ECMO-treated patients, the SNAP-II score was significantly associated with cognition and motor function at 12 months. At 24 months, low SES and non-Dutch ethnicity were associated with poor cognition, and low SES and LoS were associated with poor motor function (online suppl. table 3a). In Rome, longer LoS was significantly associated with cognition at 12 months (B -13.7; 95\% CI: -21.2 to -6.2 ) and 24 months (B -10.2 ; $95 \%$ CI: -20.0 to -0.4 , and longer LoS and motor function at 12 months (B -9.5; 95\% CI: -17.7 to -1.4$)$ and 24 months (B -16.7; 95\% CI: -25.1 to -8.2 ).

\section{Discussion}

This longitudinal study was performed in two European high-volume centers that use the same neonatal treatment protocol [1] in high-risk $\mathrm{CDH}$ patients. We found normal cognition in $78 \%$ of children aged 12 months in Rotterdam and $95 \%$ in Rome. At 24 months this was $71 \%$ in Rotterdam and $97 \%$ in Rome. Normal development of motor function occurred in $64 \%$ in Rot- terdam and $82 \%$ in Rome at 12 months. At 24 months, this was $46 \%$ in Rotterdam and $90 \%$ in Rome.

A few cross-sectional studies have reported neurodevelopmental outcome in CDH patients [8-10, 19]. Danzer et al. [9] reported delayed cognition in $32 \%$ of 41 children, assessed with BSID-II or BSID-III at a median age of 24 months, and $24 \%$ of 42 prospectively enrolled patients at 2-4 years assessed with BSID-III [8]. The prevalence of cognitive problems using similar outcome categories was comparable for Rotterdam, whilst fewer patients in Rome had cognitive delays. It is possible that patients in the study by Danzer et al. [8] were more severely ill since they were ventilated longer and more infants received ECMO. In a multicenter study by Wynn et al. [19], mean cognitive scores - obtained with BSIDIII in $48 \mathrm{CDH}$ patients at 24 months - were significantly below normal (mean 93; SD 15). We found the same trend; although different BSID versions were applied, results from Rotterdam are comparable and results from Rome are better. Many factors may contribute to cognitive delay in $\mathrm{CDH}$. Consistent with previous studies, low SES was a significant determinant of cognitive delay [19]. Previously reported predictive factors, such as tube feeding at discharge and ECMO need [19], were not confirmed in our study. However, failure to reach statistical significance for ECMO need may have been due to the fact that 6 survivors needed ECMO treatment.

In general, impaired motor function is more frequent than cognitive problems in $\mathrm{CDH}$. Our results on motor function were more favorable than those of Danzer et al. [9], who reported mild (23\%) and severe (31\%) delay on 
Table 3. Determinants of impaired neurodevelopmental outcome; results of linear regression models

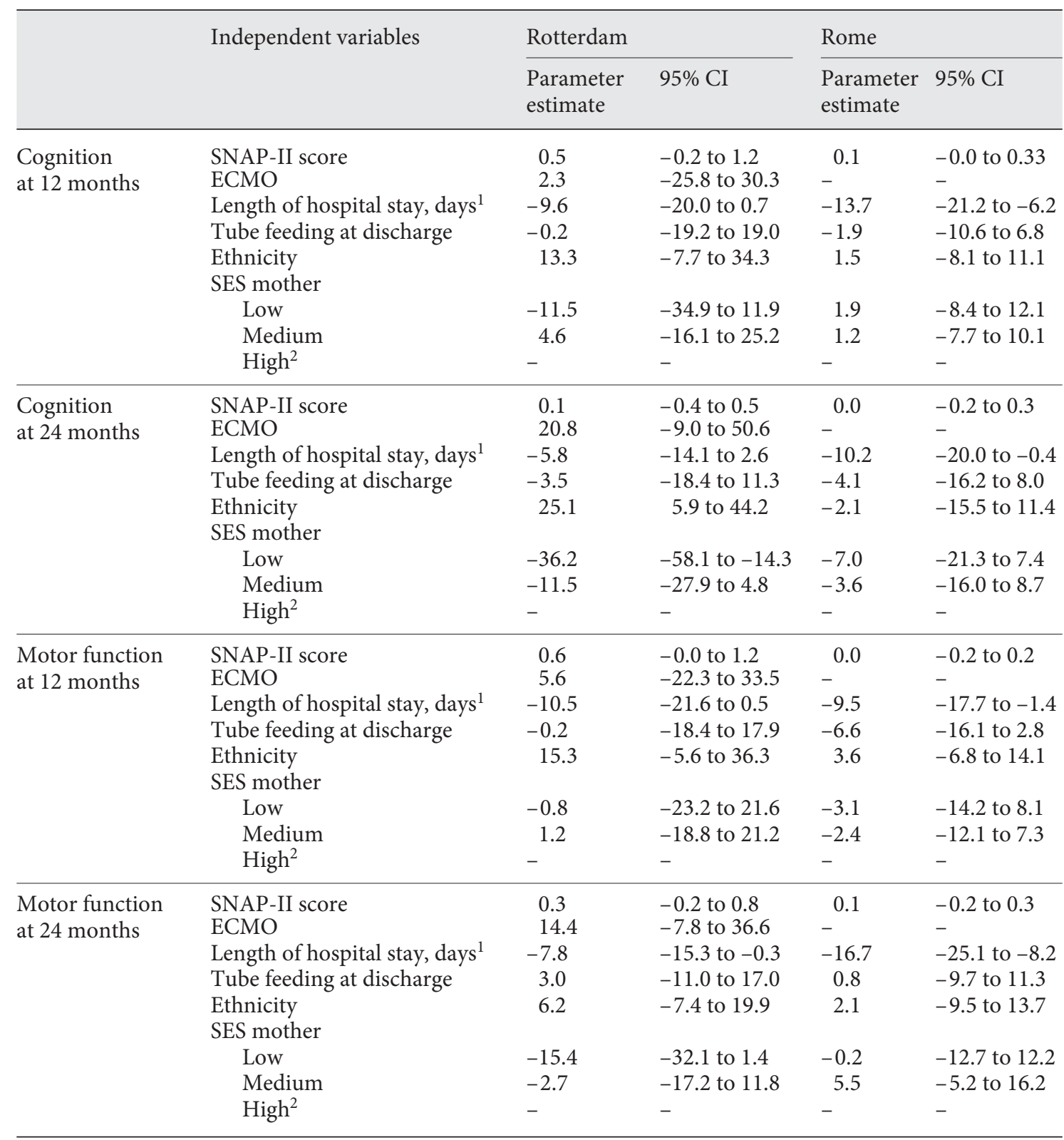

SNAP-II score $=$ Score for Neonatal Acute Physiology II. ${ }^{1}$ Because of skewed distribution, these variables were log-transformed before including them into the linear regression model. ${ }^{2}$ Reference category.

motor function at 24 months. Friedman et al. [3] reported motor problems in $60 \%$ at 1 year and $73 \%$ at 3 years. They retrospectively evaluated medical records documenting motor function. It is conceivable that standardized developmental assessment would have given other outcomes. On the other hand, Wynn et al. [19] found that motor function at 24 months was sig- nificantly lower than the population norm (mean 95; SD 11), which is comparable to Rome. Scores in Rotterdam, however, were on average 12 points lower. We could not confirm their results of adverse motor function in patients with low SES, but could confirm the result of another study [4] that LoS was associated with poorer motor function. 
The strengths of our study were that children were treated according to a standardized protocol [1] and followed in a prospective, longitudinal, standardized followup program. We collected data on ethnicity and SES, which are well-known modifiers of neurodevelopmental outcome [19].

Neurodevelopmental outcomes were different between the centers, and changes between the two measurement moments were only seen in Rome. We suggest three possible reasons. The first is the use of different assessment instruments. Since recent studies reported that BSID-II and BSID-III are not comparable [20], we refrained from pooling the data [1]. In the transition from BSID-II to BSID-III, 23 items from the BSID-II mental scale were moved to the BSID-III fine motor scale. Thus, with a higher proportion of items in the fine motor function domain in BSID-III compared to BSID-II (48 vs. $28 \%$, respectively) the fine motor score contributes relatively much to the total motor composite score in BSIDIII. This is reflected by the fact that in Rome gross motor scores are lower than fine motor scores at both ages. We assume that the outcome for patients who have a developmental delay in both cognition and motor function will not be very different for the two BSID versions. However, results may differ for patients with $\mathrm{CDH}$, who will typically show impaired motor function. Moreover, recent studies showed differences in reference scores of BSID-III between populations $[21,22]$. These considerations possibly explain the underestimation of neurodevelopmental delay in Rome. We have few standardized neurodevelopmental tests with population-matched reference data and the best option seems to compare neurodevelopmental outcome with healthy reference populations in each country. The use of American reference data in Rome might also have caused bias. In another study, neurodevelopmental outcomes were not different from those of healthy matched controls aged 12 and 36 months [10]. Disease-determining parameters were not reported, which precludes comparison with our study.

Second, professional background may have played a role. In Rotterdam, motor function at 24 months was assessed by a pediatric physical therapist, whereas in Rome assessments were performed by developmental psychologists. Since results of our study did not significantly differ from the PDI assessed by developmental psychologists in a previous study of our group [11], we assume that this may not fully explain differences in motor function. Moreover, this contradicts our assumption that neurodevelopmental outcome has worsened due to survival of severely ill patients [2].
Third, center-specific differences may have played a role. Although both tertiary intensive care centers have a comparable referral area, patients from Rotterdam may have been more critically ill than those from Rome (higher proportion of large diaphragmatic defects and higher patch use). However, SNAP-II scores and observed to expected lung-head ratio were not significantly different. The surgical approach differed, too. Primarily, a thoracoscopic approach was performed in more than $50 \%$ in Rotterdam versus open surgery in all patients in Rome. A randomized controlled trial of open versus thoracoscopic repair in $\mathrm{CDH}$ concluded that thoracoscopic repair was associated with more prolonged and severe intraoperative hypercapnia and acidosis than open surgery [23]. Moreover, only in Rotterdam was ECMO available. Since only 6 survivors received ECMO and patient characteristics did not significantly change after exclusion of these children, we assume that this hardly contributes to the outcome differences. The influence of center-specific differences such as surgical care on neurodevelopmental outcome remains speculative, but these observations emphasize the need for multicenter studies with standardized protocols. Since scores in Rotterdam slowly deteriorate at 24 months, and it is known that early adverse development in children with major congenital anomalies is predictive of development at 5 years [5], follow-up was planned for patients from Rotterdam.

Next to the differences in study design, the fact that not every child was tested at both time points could be considered a limitation. However, the numbers of dropouts were relatively low and the use of linear mixed models in the analysis over time accounted for missing data, provided that these outcomes were missing at random [24].

Interpreting outcomes in the context of international multicenter studies is difficult if the follow-up program is not standardized. International guidelines on standardization of follow-up programs in $\mathrm{CDH}$, however, are still lacking. So far, the international $\mathrm{CDH}$ registry [25] collects only prenatal, perinatal and early postnatal data. We recommend setting up standardized follow-up programs using population-appropriate reference data and similar assessment instruments as well as assessments performed by professionals of the same background. In multicenter collaboration, video assessments should be included in the training sessions. We like to make an appeal for not only international standardization of postnatal care, but also for follow-up care beyond the neonatal period, as this is essential to improve outcome for $\mathrm{CDH}$ patients. This would require standardization of treatment protocols 
and decision flowcharts of referring criteria to pediatric physical therapists/speech-language pathologists should be involved. Moreover, children should be followed up to adolescence since deficits may worsen.

In conclusion, although most $\mathrm{CDH}$ patients have normal neurodevelopment within the first 2 years of life, they are at risk for impaired motor function. Standardization of multicenter long-term follow-up programs using standardized assessment instruments and stratification to illness severity is necessary to compare neurodevelopmental outcomes between centers and to evaluate long-term effects of interventions.

\section{References}

1 Reiss I, Schaible T, van den Hout L, Capolupo I, Allegaert K, van Heijst A, Gorett Silva M, Greenough A, Tibboel D; CHE EURO Consortium: Standardized postnatal management of infants with congenital diaphragmatic hernia in europe: The CDH EURO Consortium consensus. Neonatology 2010;98:354-364.

2 van den Hout L, Schaible T, Cohen-Overbeek TE, Hop W, Siemer J, van de Ven K, Wessel L, Tibboel D, Reiss I: Actual outcome in infants with congenital diaphragmatic hernia: the role of a standardized postnatal treatment protocol. Fetal Diagn Ther 2011;29:55-63.

3 Friedman S, Chen C, Chapman JS, Jeruss S, Terrin N, Tighiouart H, Parsons SK, Wilson JM: Neurodevelopmental outcomes of congenital diaphragmatic hernia survivors followed in a multidisciplinary clinic at ages 1 and 3. J Pediatr Surg 2008;43:1035-1043.

4 Danzer E, Gerdes M, D’Agostino JA, Hoffman C, Bernbaum J, Bebbington MW, Siegle J, Sulkowski J, Rintoul NE, Flake AW, Scott Adzick N, Hedrick HL: Longitudinal neurodevelopmental and neuromotor outcome in congenital diaphragmatic hernia patients in the first 3 years of life. J Perinatol 2013;33: 893-898.

5 Mazer P, Gischler SJ, Van der Cammen-Van Zijp MH, Tibboel D, Bax NM, IJsselstijn H, Van Dijk M, Duivenvoorden HJ: Early developmental assessment of children with major non-cardiac congenital anomalies predicts development at the age of 5 years. Dev Med Child Neurol 2010;52:1154-1159.

6 Spoel M, van der Cammen-van Zijp MH, Hop WC, Tibboel D, de Jongste JC, IJsselstijn H: Lung function in young adults with congenital diaphragmatic hernia; a longitudinal evaluation. Pediatr Pulmonol 2013;48:130-137.

7 Peetsold MG, Kneepkens CM, Heij HA, IJsselstijn H, Tibboel D, Gemke RJ: Congenital diaphragmatic hernia: long-term risk of gastroesophageal reflux disease. J Pediatr Gastroenterol Nutr 2010;51:448-453.

8 Danzer E, Gerdes M, D’Agostino JA, Partridge EA, Hoffman-Craven $\mathrm{CH}$, Bernbaum J, Rintoul NE, Flake AW, Adzick NS, Hedrick HL: Preschool neurological assessment in congenital diaphragmatic hernia survivors: outcome and perinatal factors associated with neurodevelopmental impairment. Early Hum Dev 2013;89:393-400.

9 Danzer E, Gerdes M, Bernbaum J, D’Agostino J, Bebbington MW, Siegle J, Hoffman C, Rintoul NE, Flake AW, Adzick NS, Hedrick HL: Neurodevelopmental outcome of infants with congenital diaphragmatic hernia prospectively enrolled in an interdisciplinary follow-up program. J Pediatr Surg 2010;45:1759-1766.

10 Leeuwen L, Walker K, Halliday R, Fitzgerald DA: Neurodevelopmental outcome in congenital diaphragmatic hernia survivors during the first three years. Early Hum Dev 2014; 90:413-415.

11 Gischler SJ, Mazer P, Duivenvoorden HJ, van Dijk M, Bax NM, Hazebroek FW, Tibboel D: Interdisciplinary structural follow-up of surgical newborns: a prospective evaluation. J Pediatr Surg 2009;44:1382-1389.

12 Valfre L, Braguglia A, Conforti A, Morini F, Trucchi A, Iacobelli BD, Nahom A, Chukhlantseva N, Dotta A, Corchia C, Bagolan P: Long term follow-up in high-risk congenital diaphragmatic hernia survivors: patching the diaphragm affects the outcome. J Pediatr Surg 2011;46:52-56.

13 Ruiter SSH, van der Meulen B, Nakken H: The BSID-II NL: construction, standardisation, and instrumental utility. Neth J Psychol 2008; 64:15-40.

14 Bayley N: Bayley Scales of Infant and Toddler Development (BSID-III), ed 3. San Antonio, Harcourt Assessment, 2006.

15 Jani J, Nicolaides KH, Keller RL, Benachi A, Peralta CF, Favre R, Moreno O, Tibboel D, Lipitz S, Eggink A, Vaast $P$, Allegaert K, Harrison M, Deprest J; Antenatal-CDH-Registry Group: Observed to expected lung area to head circumference ratio in the prediction of survival in fetuses with isolated diaphragmatic hernia. Ultrasound Obstet Gynecol 2007;30:67-71.

16 Richardson DK, Corcoran JD, Escobar GJ, Lee SK: SNAP-II and SNAPPE-II: simplified newborn illness severity and mortality risk scores. J Pediatr 2001;138:92-100.

17 Jobe AH, Bancalari E: Bronchopulmonary dysplasia. Am J Respir Crit Care Med 2001; 163:1723-1729.
18 Centraal Bureau voor de Statistiek (Statistics Netherlands) Standaard Onderwijsindeling 2006 (The Dutch Standard Classification of Education). www.cbs.nl/nl-NL/menu/methoden/classificaties/overzicht/soi/2003/default.htm (accessed on January 6, 2015).

19 Wynn J, Aspelund G, Zygmunt A, Stolar CJ, Mychaliska G, Butcher J, Lim FY, Gratton T, Potoka D, Brennan K, Azarow K, Jackson B, Needelman H, Crombleholme T, Zhang Y, Duong J, Arkovitz MS, Chung WK, Farkouh C: Developmental outcomes of children with congenital diaphragmatic hernia: a multicenter prospective study. J Pediatr Surg 2013; 48:1995-2004.

20 Lowe JR, Erickson SJ, Schrader R, Duncan AF: Comparison of the Bayley II Mental Developmental Index and the Bayley III Cognitive Scale: are we measuring the same thing? Acta Paediatr 2012;101:e55-e58.

21 Anderson PJ, De Luca CR, Hutchinson E, Roberts G, Doyle LW; Victorian Infant Collaborative Group: Underestimation of developmental delay by the new BAYLEY-III Scale. Arch Pediatr Adolesc Med 2010;164: 352-356.

22 Chinta S, Walker K, Halliday R, LoughranFowlds A, Badawi N: A comparison of the performance of healthy Australian 3-yearolds with the standardised norms of the Bayley Scales of Infant and Toddler Development (version-III). Arch Dis Child 2014;99:621624 .

23 Bishay M, Giacomello L, Retrosi G, Thyoka M, Garriboli M, Brierley J, Harding L, Scuplak S, Cross KM, Curry JI, Kiely EM, De Coppi P, Eaton S, Pierro A: Hypercapnia and acidosis during open and thoracoscopic repair of congenital diaphragmatic hernia and esophageal atresia: results of a pilot randomized controlled trial. Ann Surg 2013;258:895-900.

24 Cnaan A, Laird NM, Slasor P: Using the general linear mixed model to analyse unbalanced repeated measures and longitudinal data. Stat Med 1997;16:2349-2380.

25 Harting MT, Lally KP: The congenital diaphragmatic hernia study group registry update. Semin Fetal Neonatal Med 2014;19:370 375 . 